\title{
On the interaction between memory scanning and response set*
}

\author{
MARIANNE W. KRISTOFFERSON \\ Department of Psychiatry, McMaster University, Hamilton, Ontario, Canada
}

\begin{abstract}
In the first of two item recognition experiments, a study by Egeth, Marcus, and Bevan (1972) was duplicated, but using a within-Ss design. Consistent with their findings, analogous differences obtained between mean RTs to positive set sizes of one and three were found to be significantly greater when Ss pressed two different keys to indicate whether or not a test letter was a member of the positive set (two-response condition) than when Ss pressed a single key to indicate only that a test letter was a positive set member (one-response condition). The number of set sizes used and results obtained for the two-response condition, which were inconsistent with characterization of the search process as either serial and exhaustive or serial and self-terminating, both limited and complicated interpretation of the obtained interaction. Further, these results suggested that the procedures used differed in important ways from those known to consistently produce linear and parallel item recognition functions for both positive and negative trials for the two-response condition. In Experiment II, those item recognition procedures which are known to allow serial exhaustive search to occur were used, and data were collected for four positive set sizes. Reaction time was a linear function of set size for both response conditions, the slopes of the functions did not differ significantly, and absolute slope values were consistent with previously reported findings for the two-response condition and letter stimuli. It is concluded that response requirements sometimes have effects on memory scanning (the slope of the item recognition function). Such an effect was observed when the experimental conditions were such as to allow neither serial and exhaustive nor serial and self-terminating memory search to occur. But, response requirements do not always have effects on memory scanning, and this was found to be the case when the experimental conditions were such as to allow serial and exhaustive memory search to occur and it was shown to occur.
\end{abstract}

It has been reported by Egeth, Marcus, and Bevan (1972) that manipulation of the response requirements in the item recognition task markedly alters the effect of the number of items in the positive set on item recognition time (the set size effect). The experiments reported in the present paper investigate the effects of response requirements on the set size effect for two different sets of item recognition procedures.

Usually, the task of $S s$ in item recognition experiments has been that of responding "yes" or "no," by pressing the appropriate one of two keys, to indicate whether a visually presented test item is or is not a member of a predefined positive set of items (two-response condition). Egeth et al compared set size effects obtained when Ss performed under the two-response condition with those obtained when the task required Ss to respond by pressing a single key, and then only when the test item was a member of a predefined positive set of items (one-response condition). In the one-response condition, Ss make no response when the test item is not a member of the positive set. Further, the effects of response set on the set size effect were investigated for two different positive set structures: "natural" and "unnatural." For both positive set structures, data were collected for positive set sizes of one and three. "Natural" sets were composed of the

*This study was supported by a grant from the National Research Council of Canada, NRC A8620. The author wishes to thank Rose Silbert and Nancy Silbert for their assistance in data collection and analysis. digit "1" when the set size was one and the digits " 1,2 , 3 " when the set size was three. "Unnatural" sets were composed of the digits " 1 " and " $1,4,7$." In all cases, the negative set of items was the complement of the positive set.

A strict between-Ss design was used. Thus, a given $S$ worked with only one response condition, one positive set structure, and one positive set size. Since for the set size of one no difference existed between the "natural" and "unnatural" conditions, six different groups (composed of four Ss each for the two-response conditions and six Ss each for the one-response conditions) participated in the experiment.

Slopes of the functions relating reaction time (RT) and positive set size obtained under the "natural" condition were $35 \mathrm{msec}$ and $2 \mathrm{msec}$ for the two-response and one-response conditions, respectively. For the unnatural condition, slopes of $45 \mathrm{msec}$ and $24 \mathrm{msec}$ were obtained for the two-response and the one-response conditions, respectively.

Based on the observation that the set size effect was greater for the two-response condition than for the one-response condition for both natural and unnatural conditions, Egeth et al concluded that there is an interaction between the set size effect and response requirements. It was also noted that, under the one-response condition and when natural sets were used, positive set size was without significant effect on item recognition time. It was concluded from this finding that either (1) "parallel processing, as indicated by the virtual 
invariance of RT with set size, can occur only when elements in the target set are related in a well-learned known way and when the response is a simple one," or (2) "processing of the elements in memory was parallel throughout, with differences in slopes reflecting differences in processing capacity available for character classification under the various experimental conditions."

Both the conclusion regarding the interaction between the set size effect and response requirements and the interpretive weight given to the absolute value of the difference between mean response time to a set size of three and a set size of one for natural sets appear to be premature. There is no way of knowing from the data analyses reported whether either (1) differences between slope values obtained from the one- and two-response conditions within each positive set structure condition are significant or (2) differences within a given response condition found between slopes for natural and unnatrual sets are significant. It is well known that there are large individual differences in response latencies. In a between-Ss design such as this, and where few Ss compose each group, it is questionable whether the absolute value of the difference between response times to a set size of three and a set size of one can be accepted at face value.

In Experiment I the design and procedures of the Egeth et al study were duplicated as closely as possible with a larger number of Ss and using a within-Ss design, except for the factor of positive set structure. That is, a given $\mathrm{S}$ worked only with natural or unnatural sets, but data were collected from each $S$ for positive set sizes of one and three, and each of these under both the one-response and the two-response conditions.

\section{EXPERIMENT I}

\section{Method}

Apparatus and Stimulus Materials. A Scientific Prototype three-field tachistoscope was used for stimulus presentation and control of trial events. The stimuli were the digits 0 through 9 , printed in black ink and centered on individual $5 \times 7$ in. white cards. The overall height of the digits was 1 in.; the visual angle subtended was approximately $1.25 \mathrm{deg}$.

Design. Each of the 24 Ss was individually tested in two experimental sessions run on consecutive days. The overall design of the experiment is shown in Table 1.

For all Ss there were four parts to each session. In two parts, the positive set size was one and, in the other two parts, the positive set size was three. Each part consisted of 48 test trials blocked into two groups of 24 trials, and each part was preceded by nine practice trials. There were 1 -min breaks between blocks and 2 -min breaks between parts. Within each of the four $S$ groups, a different one of the six possible orderings (1-3-3-1, $3-1-1-3,1-3-1-3,3-1-3-1,1-1-3-3,3-3-1-1)$ of the positive set size conditions was assigned to each of the six Ss for Session 1. In Session 2, the order assigned to each $S$ was the mirror image of that Ss order for Session 1.

Assignment of Stimuli to Positive and Negative Sets. As in the Egeth et al experiment, natural sets were made up of the digits "1" and " $1,2,3$ " and unnatural sets of the digits "1" and " 1,4 , 7." For all Ss and all conditions, the negative set was composed of all the digits $0-9$ which were not in the current positive set.
Thus, when the positive set size was one, the negative set contained nine digits; when the positive set size was three, the negative set contained seven digits.

Trial Events. A white card with a centered black dot, which served both as a warning signal and a fixation point, was displayed for $1.50 \mathrm{sec}$. A digit was exposed for $150 \mathrm{~m} \mathrm{sec}$ and a blank white card was then presented for the remainder of the 10 -sec intertrial interval. Under the two-response condition, the $S$ was instructed to respond by pressing the appropriate one of the two telegraph keys to indicate whether the test digit was or was not a member of the previously defined positive set of digits. Under the one-response condition, the $\mathrm{S}$ was instructed to respond by pressing the single telegraph key to indicate that the test digit was a member of the previously defined positive set of digits. All Ss were instructed to use their preferred hand for the positive response under both response conditions and their nonpreferred hand for the negative response under the two-response condition. The time from the onset of the test digit to the Ss response was measured with an accuracy of $\pm 1 \mathrm{msec}$ and was recorded by $E$. Auditory feedback informed the $S$ as to the correctness of her response.

Test Stimulus Sequence. For each $\mathrm{S}$, each session, and each part of each session, a different random ordering of positive and negative trials was used. Within each part of the experiment, $50 \%$ of the trials required a positive response. When the positive set contained more than one digit, each digit in the set was presented randomly but equally often. The digits making up the negative sets were also randomly distributed, with as nearly equal occurrences as possible.

Subjects and Instructions. The $24 \mathrm{Ss}$ were paid McMaster University female student volunteers who were naive to the experimental tasks. Before each part of each session, the $\mathrm{S}$ was shown the digits composing the positive set. All Ss were encouraged to respond as rapidly as they could without making errors.

\section{Results}

For each $\mathrm{S}$ and for each response condition separately, the slope of the linear function relating response latency and positive set size was calculated for positive trials (trials requiring a "yes" response). Differences between slope values obtained under the one-response and two-response conditions were calculated for each S. In order to determine whether order of response conditions significantly affected differences obtained between slope values for the two response conditions, $t$ tests were run (1) between difference scores obtained by the Ss in Group 1 and the Ss in Group 2 (see Table 1) and (2) between difference scores obtained by the Ss in Group 3 and the Ss in Group 4. Values of $t$ in both cases were found to be $<1$. Since order of response conditions was found to be without significant effect on differences obtained

Table 1

Design of Experiment I

\begin{tabular}{ccccc}
\hline & & $\begin{array}{c}\text { Positive Set } \\
\text { Structure } \\
\text { (Sessions }\end{array}$ & \multicolumn{2}{c}{ Response Condition } \\
\hline 1 & 6 & Natural & One-Response & Two-Response \\
2 & 6 & Natural & Two-Response & One-Response \\
3 & 6 & Unnatural & One-Response & Two-Response \\
4 & 6 & Unnatural & Two-Response & One-Response \\
\hline
\end{tabular}


between slope values calculated under the different response conditions for either "natural" or "unnatural" sets, for all subsequent analyses data obtained from the 12 Ss who worked with natural sets were combined and data obtained from the 12 Ss who worked with unnatural sets were combined.

Response time is shown plotted as a function of set size for Ss who worked with natural sets in the left side of Fig. 1. Data are plotted separately for the one-response condition, the two-response condition positive trials, and the two-response condition negative trials. Analogous functions are shown for Ss who worked with unnatural sets in the right side of the same figure. Without exception, mean response latencies to the set size of three were found to be significantly $(p<.01)$ greater than to the set size of one. Values of $t(d f=11)$ obtained for natural sets were $8.50,10.01$, and 5.11 for the one-response condition, the two-response condition positive trials, and the two-response condition negative trials, respectively. Analogous values of $\mathrm{t}(\mathrm{df}=11)$ obtained for unnatural sets were $5.85,12.69$, and 4.77 .

In order to determine the effect of positive set structure on the set size effect, comparisons between groups were made using $t$ tests for unmatched samples. Slope values obtained under the one-response condition for natural (mean $=24.3 \mathrm{msec}$ ) and unnatural (mean = $24.5 \mathrm{msec}$ ) were found not to differ significantly $(\mathrm{t}<1)$. Slope values obtained from positive responses under the two-response conditions for natural (mean $=33.1 \mathrm{msec}$ ) and unnatural (mean $=40.0 \mathrm{msec}$ ) were also found not to differ significantly $(\mathrm{t}=1.58, \mathrm{df}=22, \mathrm{p}>.10)$. However, slope values obtained from negative trials under the two-response condition were found to be significantly greater $(t=2.21, d f=22, p<.05)$ for unnatural $($ mean $=30.2 \mathrm{msec}$ ) than natural (mean $=$ $20.4 \mathrm{msec}$ ).

Within-group $t$ tests were run to determine whether set size effects obtained under the different response conditions differed significantly for Ss assigned to the natural sets. Slope values obtained under the two-response condition, when calculated from positive trials only, were found to be significantly $(\mathrm{t}=2.53$, $\mathrm{df}=11, \quad \mathrm{p}<.05>.02)$ greater than slope values obtained under the one-response condition. Slope values obtained from positive trials under the two-response condition were significantly $(t=4.10, d f=11, p<.01)$ greater than slope values obtained from negative trials under the two-response condition. And slope values obtained from negative trials under the two-response condition did not differ significantly $(t<1)$ from slope values obtained under the one-response condition.

A very similar pattern of results was obtained when $t$ tests were run between slope values obtained under the different response conditions for the group of Ss who worked with unnatural sets. Slope values obtained from positive trials under the two-response condition were

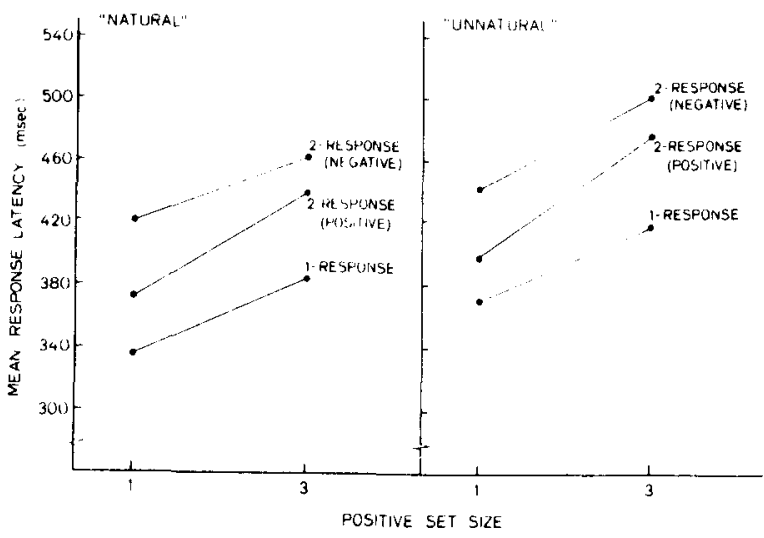

Fig. 1. Mean response latency as a function of positive set size for one- and two-response conditions and each of these for both natural and unnatural positive set constructions. Positive and negative trials data are shown separately for the two-response condition.

found to be significantly $(t=2.56, \quad d f=11$, $p<.05>.02)$ greater than slope values obtained under the one-response condition. Slope values obtained from the positive trials under the two-response condition were significantly greater $(\mathrm{t}=3.18, \mathrm{df}=11, \mathrm{p}<.01)$ than slope values obtained from negative trials under the two-response condition. And, finally, slope values obtained from negative trials under the two-response condition did not differ significantly $(t=1.46, d f=11$, $p>.10)$ from slope values obtained under the one-response condition.

Error levels were low in all conditions and never exceeded $2.3 \%$ for any positive set size.

\section{Discussion}

The differential effects of natural and unnatural sets on the set size effect reported by Egeth et al were not obtained in this experiment. A significant positive set size effect was found for both natural and unnatural sets for both one- and two-response conditions. Further, the set size effect obtained was not significantly different for natural and unnatural sets for either of the response conditions when slopes calculated from positive response trials were compared. Only when slopes obtained from negative trials under the two-response condition were compared was a significantly greater set size effect found for unnatural than for natural sets.

The results obtained regarding the effect of the response requirements on the set size effect in item recognition performance are partially consistent with the Egeth et al findings. That is, for both natural and unnatural sets, the positive set size effect was found to be significantly greater for the two-response condition than for the one-response condition when "yes" or positive RTs were compared. The interpretation of this result is complicated by the finding that for both natural and unnatural sets slope values determined from negative 
RT trials in the two-response condition were found not to differ significantly from slope values obtained under the one-response condition. And, further, the findings of greatest statistical significance were differences obtained between slope values calculated from positive trials and slope values calculated from negative trials in the two-response condition. These comparisons show the set size effect to be significantly less for negative than for positive trials for both natural and unnatural sets.

Because only two positive set sizes were used in this experiment, as in the Egeth et al experiments, it is not possible to specify the Response Set by Set Size Effect interaction further. It may be that the form of the function relating response latency and positive set size is altered when the response requirements are changed. If such were the case, it would suggest that the process underlying the set size effect is different in kind depending upon the response requirements of the task. On the other hand, it may be that the item recognition function is the same under both response conditions but the slope is significantly less under the one-response condition. If this were the case, it would suggest that the process underlying the set size effect is the same in kind for both response conditions but that the process is accelerated under the one-response condition.

While these data are insufficient to allow description of the functions relating item recognition time and positive set size, one feature of the two-response condition data that is important to any inference about the process underlying the set size effect departs markedly from what has usually been reported when the typical set of item recognition procedures is used. That is, in the two-response condition, slopes from negative trials were found to be significantly less than slopes from positive trials. This finding, for example, rules out characterization of the memory scanning process as either serial and exhaustive or serial and self-terminating, since to infer the first requires equality of positive and negative slopes and to infer the second requires negative slope values to be double positive slope values.

It has been shown (Kristofferson, 1972b; Ross, 1970) for the two-response condition that the nature and absolute value of the set size effect depends importantly on such details of procedure as (1) whether or not for a given $S$ the same item sometimes requires a positive response and sometimes a negative one (response consistency vs response consistency) and (2) on this variable in combination with whether or not the positive sets are constructed so that each positive set contains all the items also contained in smaller positive sets (nested vs nonnested positive sets).

Item recognition functions are typically found to be linear and to have the same slope values for positive and negative trials when for a given $S$ positive sets are nonnested, change from session to session, and when the same item sometimes requires a positive response and sometimes a negative one (response inconsistency) (e.g.,
Sternberg, 1966; Chase \& Calfee, 1969; Sternberg, 1969). Further, under these conditions the set size effect has been found to remain stable over a course of prolonged practice (Kristofferson, 1972a).

However, when for a given $\mathrm{S}$ positive sets are nested, constant over all sessions, and when response consistency is present, the item recognition function is found to be negatively accelerated (Ross, 1970; Kristofferson, 1972b) and the effect of set size is significantly greater for positive than for negative trials (Kristofferson, 1972b). Also, under these conditions, the set size effect has been shown to decrease with practice (Kristofferson, 1972b; Ross, 1970).

In Experiment $I$ a condition of nested positive sets was combined with a condition of partial response consistency. The digit " 1 ," common to both positive set sizes, always required a positive response, while the digits " 2 " and " 3 " under the natural set structure condition and " 4 " and " 7 " under the unnatural set structure condition required a negative response when the set size was one and a positive response when the set size was three. Just as the effects of prolonged practice on the set size effect have been found to be different for different procedural variations of the task, the response requirements of the task may also have differential effects on the set size effect for different variations of item recognition procedures. Therefore, rather than continue to investigate the effect of response requirements on the set size effect in a "hybrid" item recognition procedure, such as that used in Experiment I, it appeared the most informative approach would be to investigate the effect of response requirements on the set size effect where enough positive set sizes were used to allow description of the item recognition function and using those procedures which have yielded consistent evidence of linear functions and equality of positive and negative slope values under the two-response condition.

\section{EXPERIMENT II}

\section{Method}

The apparatus, stimulus materials, trial events, and test stimulus sequences were the same as in Experiment I. McMaster University student paid volunteers (12 males and 12 females), who were naive to the experimental task, served as Ss. Each S participated in two sessions run on consecutive days. For half of the Ss (six males and six females), data were collected in Session 1 under the one-response condition and in Session 2 under the two-response condition. For the remaining Ss, the order of response conditions was reversed.

For each $S$ each session consisted of four parts, one for each of the positive set sizes one, two, three, and four. A different one of the 24 possible orderings of the four positive set sizes was randomly assigned to the $24 \mathrm{Ss}$ for each session separately. Trials were grouped into blocks of 24 ; there were two consecutive blocks per set size within each session. A 1-min break occurred between blocks and a 3-min break between conditions. Nine practice trials preceded each part.

Immediately prior to each part, the digits composing the 


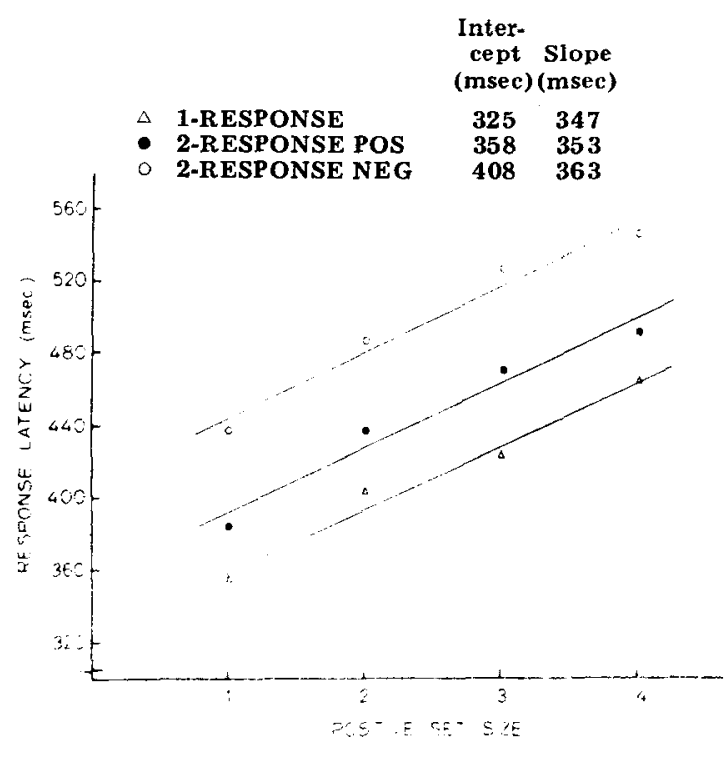

Fig. 2. Mean response latency as a function of positive set size shown separately for the one-response condition, the two-response condition positive trials, and the two-response condition negative trials. Least-squares best-fitting straight lines are drawn through each set of data, and intercept and slope values for these functions are shown.

positive set for that part were displayed sequentially in the tachistoscope. After the set of digits had been exposed, the $\mathrm{S}$ was asked to repeat the digits aloud.

Assignment of Digits to Positive and Negative Sets. For each pair of $S$ s (one $S$ in the group with the one-response condition first and one $S$ in the group with the two-response condition first) and each session separately, the $d$ igits 0 through 9 were rand omly assigned without replacement to constitute positive set sizes of one, two, three, and four. A further restriction on the assignment of digits to positive sets was that, for sets greater than one, the digits $d$ id not form a consecutive set. The negative items for each set size condition were all the digits 0 through 9 which were not members of the positive set for that particular part.

\section{Results and Discussion}

For each S set size effects or slopes were calculated from positive trials for each response condition separately and from negative trials for the two-response condition. Order of response conditions was found to have no significant effect on differences obtained between set size effects for positive trials for the two-response condition $(t<1)$. Therefore, data obtained from all 24 Ss were combined. Errors occurred very infrequently under all conditions and never occurred on more than $1.7 \%$ of the trials in any condition.

In Fig. 2 positive set size is shown plotted against response latency for data obtained under the one-response condition and the two-response condition, for which positive and negative trials data are shown separately. Best-fitting linear functions are drawn through each set of data. In all cases, the data are quite well described as linear; the percent of variance accounted for by linear regression ranged from 96.4 to 97.7. Differences in slope values obtained both between response conditions and within the two-response condition were trivial, in no case exceeding $1.65 \mathrm{msec}$. Not surprisingly, therefore, tests for significant differences between set size effects all yielded $t$ values of less than 1. Further, the absolute magnitude of the set size effect (approximately $35 \mathrm{msec}$ ) is quite representative of values usually reported for the two-response condition and the traditional item recognition paradigm. Thus, all the important features typically found to describe item recognition performance under this set of procedures were reproduced in Experiment II, for both one- and two-response conditions.

On the basis of the findings from Experiment II, it is concluded that, when the conditions of the experiment are such as to allow serial and exhaustive memory search to occur and it is shown to occur, then the set size effect obtained under the one-response condition does not differ qualitatively or quantitatively from that obtained under the two-response condition.

However, from the results of Experiment I, which were in part consistent with the findings reported by Egeth et al, it is concluded that response requirements do sometimes have a significant effect on the set size effect. Response requirements appear to have an effect when the conditions of the experiment are not such as to allow either serial and exhaustive or serial and self-terminating memory search to occur.

\section{REFERENCES}

Chase, W. G., \& Calfee, R. C. Modality and similarity effects in short-term recognition memory. Journal of Experimental Psychology, 1969, 81, 510-514.

Egeth, H., Marcus, N., \& Bevan, W. Target-set and response-set interaction: Implications for models of human information processing. Science, 1972, 176, 1447-1448.

Kristofferson, $M$. W. Effects of practice on character-classification performance. Canadian Journal of Psychology, 1972a, 26, 54-60.

Kristofferson, $M$. W. When item recognition and visual search functions are similar. Perception \& Psychophysics, 1972b, 12, 379-384.

Ross, J. Extended practice within a single-character classification task. Perception \& Psychophysics, 1970, 8, 276-278.

Sternberg, S. High-speed scanning in human memory. Science, $1966,153,652-654$.

Sternberg, $\mathbf{S}$. Memory-scanning: Mental processes revealed by reaction-time experiments. American Scientist, 1969, 57. 421-457.

(Received for publication May 6, 1974; revision accepted July $7,1974$. ) 